\title{
Detection of MET and SOX2 amplification by quantitative real-time PCR in non-small cell lung carcinoma
}

\author{
YI-RAN CAI ${ }^{1}$, HAI-QING ZHANG ${ }^{1}$, ZONG-DE ZHANG $^{2}$, JING MU $^{1}$ and ZI-HUI LI ${ }^{2}$ \\ ${ }^{1}$ Department of Pathology, Beijing Tuberculosis and Thoracic Tumor Research Institute, \\ Beijing Chest Hospital; ${ }^{2}$ Laboratory of Molecular Biology for Mycobacteria, \\ Beijing Tuberculosis and Thoracic Tumor Research Institute, Beijing 101149, P.R. China
}

Received September 1, 2010; Accepted December 9, 2010

DOI: $10.3892 / \mathrm{ol} .2010 .229$

\begin{abstract}
Non-small cell lung carcinoma is a leading cause of cancer-related death. Amplification of the two oncogenes MET and SOX2 is frequently encountered in non-small-cell lung carcinoma. This study aimed to use real-time quantitative PCR to assess the correlation of MET and SOX2 amplification with clinicopathological factors. This study was conducted using 115 tissue samples including 57 squamous cell carcinomas (SCCs), 50 adenocarcinomas (ADCs) and 8 adenosquamous carcinomas (ADSCs). A total of 67 patients $(58.3 \%$ ) had a history of smoking. Our results showed that the frequency of MET amplification in SCCs was significantly higher compared to ADCs $\left(\chi^{2}=8.0, \mathrm{P}=0.005\right)$. SOX2 showed a markedly preferential amplification in SCCs compared to ADCs in the smoking group cases $(\mathrm{P}=0.014)$. Lymph node invasion correlated with MET amplification in SCCs marginally more significantly compared to ADCs $(\mathrm{P}=0.02)$. The amplified MET occurred more frequently in SCCs compared to ADCs correlated to tumor dimension at a small scale $(<5 \mathrm{~cm})$ $(\mathrm{P}=0.01)$. No significant difference in SOX2 amplification was found with regards to lymph node metastasis or tumor dimension. SOX2 and MET amplifications were not associated with gender or age. However, MET amplification in SCCs among patients younger than 64 years of age was higher compared to ADCs and ADSCs $(\mathrm{P}=0.03)$. Among ADSCs, $M E T$ was not amplified among patients who had never been smokers or were younger than 64 years of age. Neither MET nor $S O X 2$ were amplified in tumors with dimensions $<5 \mathrm{~cm}$ and without lymph node invasion. Findings of this study showed that MET and SOX2 amplifications are more common in the SCCs of smokers. Moreover, MET amplification is intrinsic in SCCs particularly among smokers, with regards to
\end{abstract}

Correspondence to: Dr Yi-Ran Cai, Department of Pathology, Beijing Tuberculosis and Thoracic Tumor Research Institute, Beijing Chest Hospital, No. 97 Ma Chang, Tongzhou District, Beijing 101149, P.R. China

E-mail:cyr7172@gmail.com

Key words: non-small cell lung carcinoma, MET gene, SOX2 gene, real-time quantitative PCR tumor growth, lymph node invasion and negative correlation to SOX2 amplification. The incidence of discrepancy in the amplifications of MET and SOX2 in SCCs and ADCs suggests that the MET and SOX2 genes play different roles in SCC and ADC tumorigenesis, respectively, particularly among smokers.

\section{Introduction}

Histological subtypes of non-small-cell lung carcinomas (NSCLC) include adenocarcinomas (ADC), squamous cell carcinomas (SCC) and adenosquamous carcinomas (ADSC). NSCLCs represent $80 \%$ of lung cancers and are further classified into ADC, including bronchioloalveolar carcinoma (BAC), and SCC (1). Receptor tyrosine kinases (RTKs) regulate various key processes in mammalian development, cell function and tissue homeostasis. Alterations at the level of the receptor and its ligand lead to the activation of a number of signaling pathways, each of which may contribute to cancer progression. Deregulation of RTKs by mutation, gene rearrangement, gene amplification and overexpression of both receptor and ligand play a role as causative factors in the development and progression of various types of human cancer (2-4). The tyrosine-kinase epidermal growth factor receptor (EGFR) pathway has been shown to play a crucial role in the pathogenesis of NSCLC, leading to the development of targeted therapeutic agents using small molecule EGFR tyrosine kinase inhibitors (TKIs) such as gefitinib or erlotinib (5-7).

Recent clinical evidence of EGFR-TKIs in refractory and advanced NSCLCs potentially indicate that EGFR-TKIs target other deregulated growth factor signaling pathways, such as the hepatocyte growth factor (HGF)/MET pathway. A recent study showed that $M E T$ amplification leads to gefinitib secondary resistance and may also explain the resistance noted in certain patients (8). The MET gene is located on band 7q31, and encodes a transmembrane tyrosine kinase receptor for $\mathrm{HGF} / \mathrm{scatter}$ factor (SF), located on band 7q25. The MET gene is the prototypic member of a subfamily of RTKs. The MET RTK family is structurally distinct from other RTK families and is the only known high-affinity receptor for HGF, also known as SF $(9,10)$. In addition to the proliferative and antiapoptotic activities that are common to various growth factors, MET elicits unique motogenic and morphogenic effects by stimulating cell-cell detachment, migration, invasion, 
tubule formation and branching $(11,12)$. These activities of the $M E T$ signaling pathway provided examples of the mechanisms by which this pathway is involved in tumor development and progression. MET is usually considered to be an oncogene (13) and appears to play a role in ADCs. Cigarette smoking induces overexpression of HGF in type II alveolar pneumocytes and lung cancer cells (14). Overexpression of HGF in lung cancer cells induces alveolar differentiation/proliferation, and MET activation may play a crucial role in well-differentiated lung ADCs (15-17). MET amplification has already been described in gastric and esophageal cancers $(18,19)$.

The transcription factor SOX2, a member of the SRY-high mobility box transcription factor family, is expressed in epithelial cells of the foregut, including the pharynx, esophagus, trachea, bronchi and bronchioles, but is excluded from the peripheral and alveolar regions of the lung (20). SOX2 is also expressed in developing respiratory epithelium, but is restricted to the conducting airways of the mature lung (21). SOX2 is induced in the bronchiolar epithelium during repair following toxicant-induced injury (22). Overexpression of SOX2 in lung epithelium during early development disrupted branching morphogenesis, resulting in cystic lungs and neonatal death (21). It has been suggested that $S O X 2$, which belongs to group B of the SOX family, plays a critical role in cell fate determination, differentiation and proliferation $(23,24)$. A recent study showed SOX2 amplification on band 3q26.3 and intense SOX2 immunostaining in lung SCC, indicating potential active transcriptional regulation by $S O X 2$. SOX2-overexpressing lung epithelial cells and embryonic stem cells (ESCs) reveal that SOX2 contributes to the activation of ESC-like phenotypes and provides clues pertaining to the de-regulated genes involved in the malignant phenotype (25). Limited data are available with regards to the MET and SOX2 copy number in NSCLC. Our study aimed to simultaneously analyze the MET and SOX2 status in a NSCLC cohort to elucidate the potential role of the MET and SOX2 signaling pathway and to demonstrate $M E T$ and SOX2 gene amplification to its clinicopathological features.

\section{Materials and methods}

Samples. A total of 115 primary lung cancers were analyzed from Chinese patients whose surgeries were performed in Beijing Chest Hospital, China, between 2007 and 2009. Resected tumors were formalin-fixed and paraffin-embedded until the DNA was extracted. Corresponding non-malignant peripheral lung tissues were also collected. The specimens were reviewed by two reference pathologists (Hai-Qing Zhang and Yi-Ran Cai) to confirm the diagnosis and predominance $(>70 \%)$ of cancer tissue in the tumor specimens. BAC was defined as previously described (26). The two observers were blind to the patient outcomes. Clinicopathological characteristics such as age, gender, histological subtype and feature of tumors were obtained.

DNA extraction. Genomic DNA was derived from formalinfixed paraffin-embedded tumors in blocks. The tissues (50-100 mg) were scraped off the block, de-paraffinized twice in xylene, rinsed twice with absolute ethanol and washed with pure water. The tissues were suspended in $500 \mu \mathrm{l}$ of glutaral- dehyde containing 100-200 $\mu \mathrm{g}$ of proteinase $\mathrm{K}$ (Promega, Madison, WI, USA) and incubated overnight at $55^{\circ} \mathrm{C}$. Finally, the DNA was purified through columns (DNeasy Tissue Kit, Tiangen, Beijing, China) following the manufacturer's instructions. The lysis mixture was centrifuged for $1 \mathrm{~min}$ to remove undigested tissue and then the eluant (DNA) was combined with $200 \mu \mathrm{l}$ of GB buffer, vortexed and incubated at $70^{\circ} \mathrm{C}$ for $10 \mathrm{~min}$. Following the addition of $210 \mu \mathrm{l}$ of $100 \%$ ethyl alcohol, the sample was vortexed, added to a spin column and centrifuged at $9000 \mathrm{rpm}$ for $1 \mathrm{~min}$. The filtrate was discarded, $500 \mu 1$ of GD buffer was added, and the column was centrifuged at $8000 \mathrm{rpm}$ for $1 \mathrm{~min}$. The filtrate was discarded, and the preceding step was repeated twice, with the column spin being rinsed by PW buffer. The DNA was then suspended in $100 \mu 1$ of TE [10 mM Tris (pH 8) and 1 mM EDTA (pH 8)]. The DNA concentration was measured by UV absorbance at $260 \mathrm{~nm}$, and the samples were stored at $4^{\circ} \mathrm{C}$ until further use.

Real-time quantitative PCR. The Taq Man PCR reaction was used to evaluate the amplification of MET and SOX2 genes in lung cancer. This PCR reaction included a dual-labeled fluorogenic probe (5_ reporter and 3_quencher dyes) and the amount of fluorescence detected was directly proportional to the amount of DNA synthesized (27-29). The Taq Man probes and primers were designed using Primer Express software (Applied Biosystems, Warrington, UK) and were optimized according to the manufacturer's guidelines. Target gene probes (MET and SOX2) and reference gene probes (GAPDH) contained a TAMRA dye at the 3_end and FAM as a reporter dye at the 5_ end. The primer and probe sequences were: GAPDH primer (forward, 5'-TCG ACA GTC AGC CGC ATC TTC TTT-3'; reverse, 5'-ACC AAA TCC GTT GAC TCC GAC CTT-3'; and probe, 5'-6FAM-AGC CAC ATC GCT CAG ACA CCA TGG G-TAMRA-3'); MET primer (forward, 5'-TGC AGC GCG TTG ACT TAT TCA TGG-3'; reverse 5'-GAA ACC ACA ACC TGC ATG AAG CGA-3'; and 5'-6FAM-AGG AGA CCT CAC CAT AGC TAA TCT TGG G-TAMRA-3'); SOX2 primer (forward, 5'-CAC ATG AAG GAG CAC CCG GAT TAT-3'; reverse 5'-GTT CAT GTG CGC GTA ACT GTC CAT-3'; and 5'-6FAM-TGA AGA AGG ATA AGT ACA CGC TGC CC-TAMRA-3'). Monoplex real-time quantitative PCR was performed using a $50 \mathrm{ng}$ template extracted from paraffin-embedded tissues. The reactions were carried out in a total volume of $25 \mu \mathrm{l}$ containing $1 \mathrm{X}$ SsoFast probes supermix (BioRad, USA). The primer and probe concentrations were optimized for each target according to the manufacturer's instructions. The PCR program consisted of $50^{\circ} \mathrm{C}$ for $2 \mathrm{~min}$ and $95^{\circ} \mathrm{C}$ for $10 \mathrm{~min}$ followed by 40 cycles of $95^{\circ} \mathrm{C}$ for $15 \mathrm{sec}$ and $56^{\circ} \mathrm{C}$ for $1 \mathrm{~min}$. In each run, the templates were assayed in triplicate and for the majority of samples each run was repeated at least twice.

Standard curve. Real-time PCR technology is often used for the relative quantification of nucleic acids (30). The slightest impurities in the sample and uneven template fragmentation due to improper fixation severely distort absolute quantification. Consequently, a relative quantification algorithm is strongly recommended. In performing relative calculations, the distortions of the amplification efficiency due to fixation artifacts or sample impurities were eliminated. Whether PCR 


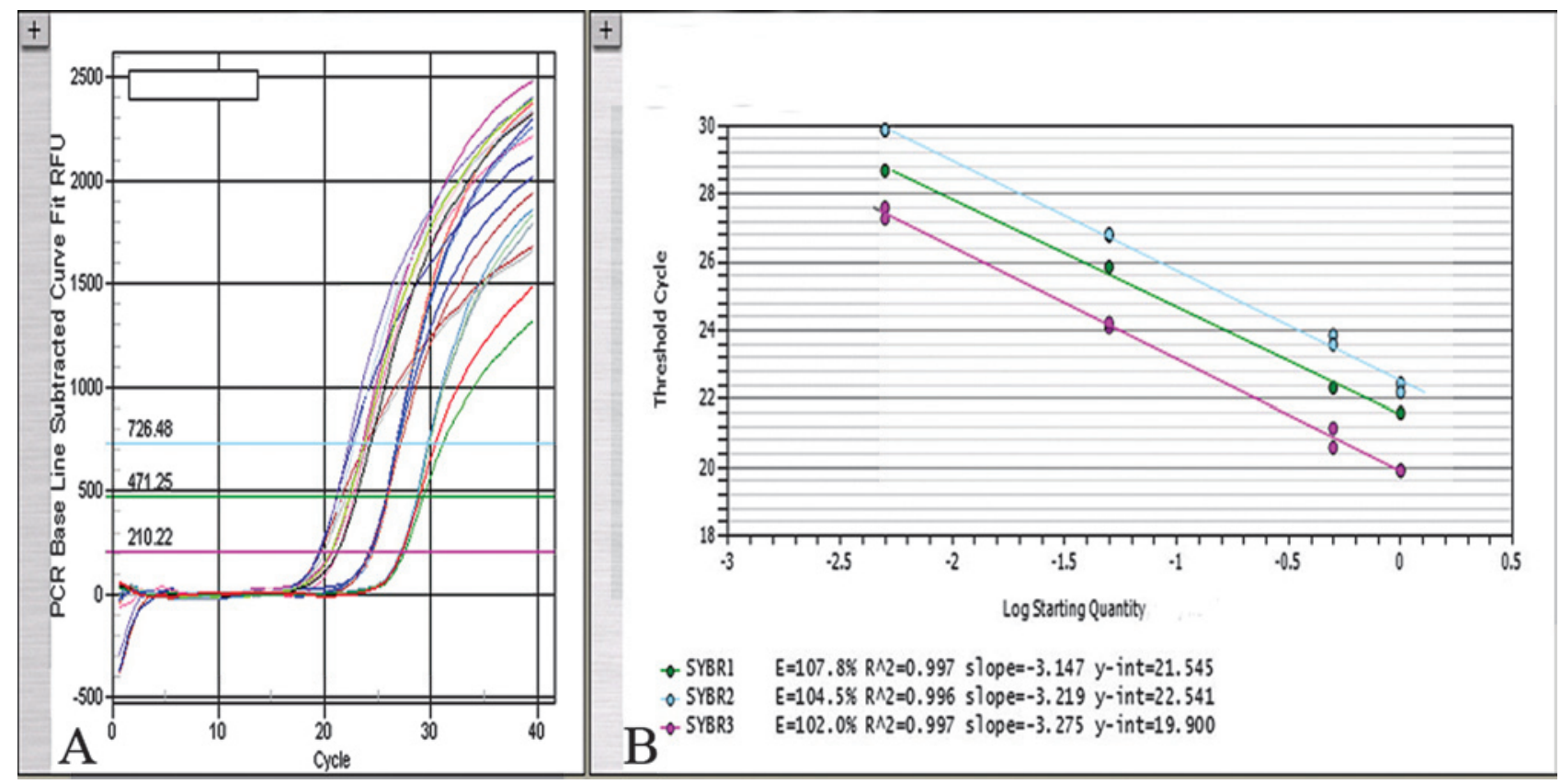

Figure 1. Taq Man GAPDH, MET and SOX2 standard curves obtained by real-time PCR applied on DNA from FFPE tissue. (A) Amplification plots for four serial dilutions of a normal DNA sample. DNA dilution increases from left to right from 0, 1:2, 1:20 to 1:200 per triplicate. (B) Standard curves plotting the $\log$ dilution $(\mathrm{x})$ against the threshold cycle numbers $(\mathrm{Ct})$. The magenta line represents the standard curve obtained for GAPDH, green line for MET and skyblue line for $S O X 2$. Regression parameters of the standard curves are displayed: E, efficiency of amplification; $\mathrm{R}^{\wedge} 2$, coefficient of correlation; $\mathrm{y}$-int, intercept.

would be quantitative when the DNA had been extracted from fixed tissues under our experimental conditions was defined. For fixed tissues, the $260 \mathrm{~nm}$ DNA concentration is not an exact reflection of the DNA quantity to be amplified, since it is altered by the presence of PCR inhibitory factors, as well as by degradation or chemical modification of the DNA due to the fixative. Consequently, a standard curve was constructed by amplifying serial dilutions (0, 1:2, 1:20, 1:200) of normal DNA from 500 to $2.5 \mathrm{ng}$ (measured at $260 \mathrm{~nm}$ ) (Fig. 1). A constant numerical ratio of target and reference genes was measured in this concentration range. The PCR efficiency in all of the cases was controlled (range 100-110\%). The standard deviation (SD) of the values was in the range of 0.5-1 cycle. Data analysis was carried out using iCycler IQ5 optical system software (version 2.0, BioRad) which calculates the threshold cycle numbers $(\mathrm{Ct})$. The $\mathrm{N}$ cut-off value for gene amplification was determined for the MET and SOX2 genes in paraffin waxembedded tissues using cumulative frequencies of the values. Individual measurements that fell outside the 97.5 percentile (the cut-off point) were considered to be outside the normal values. Only standard curves with correlation coefficients of $\geq 0.98$ were used. The copy number change of the MET and SOX2 genes in correlation to glyceraldehyde-3-phosphate dehydrogenase $(G A P D H)$ was determined using the formula $\left(\mathrm{T}_{\text {target gene }} / \mathrm{T}_{\text {GAPDH }}\right) /\left(\mathrm{N}_{\text {target gene }} / \mathrm{N}_{\text {GAPDH }}\right)$, where $\mathrm{T}_{\text {target gene }}$ and $\mathrm{T}_{\text {GAPDH }}$ were determined from sample DNA using MET, SOX2 and $G A P D H$; and the normalized ratio of MET and SOX2 was determined from a normal sample of 20 tuberculosis patients selected at random. The results of samples for increased MET and SOX 2 relative copy numbers were confirmed by repeating the experiments $\geq 3$ times. An interval for the normalized ratio values was calculated corresponding to the mean $(\mathrm{M}) \pm 2 \mathrm{SD}$.
A lung tumor sample was considered to be amplified if its ratio was $>\mathrm{M}+2 \mathrm{SD}$, or as deleted if its ratio was $<\mathrm{M}-2 \mathrm{SD}$ (31).

Statistical analysis. The associations between the categorical variables were examined using the $\chi^{2}$ or Fisher's exact tests. Statistical tests were two-sided. The significant level $\alpha=0.05$ and $\mathrm{P}<0.05$ were considered to indicate statistical significance for the cross table (Table I), but when referred to co-comparison within the cross table, the significant level was specified at $\alpha / \mathrm{n}$ ( $\mathrm{n}=$ combinations of comparison). The correlation between MET and SOX2 amplifications was evaluated by calculating the $r$ value. The statistical analysis was performed using SAS version 9.2 (SAS Institute Inc., Cary, NC, USA) for Windows.

\section{Results}

Patient characteristics and histopathological features. The clinicopathological characteristics of 115 non-small cell carcinomas are listed in Table I. The patients were primarily male $(70.4 \%, \mathrm{n}=81)$. A total of 67 patients $(58.3 \%)$ had a former or current history of smoking. The median age was 58 years (range 27-77). The patients were diagnosed with 57 SCCs (49.5\%) and 50 ADCs (43.5\%), including 8 BACs and 8 ADSCs (7\%). ADSCs, as a special substance in histological types, which exhibit ADC and SCC, were used as connectors to assess the relationship between ADC and SCC. A total of 54 patients $(47 \%)$ presented with lymph node metastasis. Of the tumors, $10(8.7 \%)$ were well differentiated, whereas $58(50.4 \%)$ exhibited moderate and $47(40.9 \%)$ exhibited poor differentiation. From a total of 57 cases in the SCC subgroup, 28 cases (49.1\%) had tumor dimensions of $<5 \mathrm{~cm}, 49$ cases $(86 \%)$ had a history of smoking and all 57 cases were moderately or poorly 
Table I. Correlation of SOX2 and MET gene amplifications with their pathological features.

\begin{tabular}{|c|c|c|c|c|c|c|c|c|}
\hline \multirow[b]{3}{*}{ Variable } & \multicolumn{4}{|c|}{ SOX2 } & \multicolumn{4}{|c|}{ MET } \\
\hline & \multicolumn{2}{|c|}{ Amplification } & \multicolumn{2}{|c|}{ Non-amplification } & \multicolumn{2}{|c|}{ Amplification } & \multicolumn{2}{|c|}{ Non-amplification } \\
\hline & Patient no. & $\%$ & Patient no. & $\mathrm{P}$ & Patient no. & $\%$ & Patient no. & P-value \\
\hline All 57 cases & 30 & 26.1 & 85 & & 13 & 11.3 & 102 & \\
\hline \multicolumn{9}{|l|}{ Gender } \\
\hline Male & 24 & 29.6 & 57 & 0.18 & 10 & 12.4 & 71 & 0.75 \\
\hline Female & 6 & 17.7 & 28 & & 3 & 8.8 & 31 & \\
\hline \multicolumn{9}{|l|}{ Age, years } \\
\hline$<64$ & 20 & 27 & 54 & 0.75 & 9 & 12.2 & 65 & 0.77 \\
\hline$\geq 64$ & 10 & 24.4 & 31 & & 4 & 9.8 & 37 & \\
\hline \multicolumn{9}{|l|}{ Histological type } \\
\hline Adenocarcinoma & 10 & 20 & 40 & 0.39 & 1 & 2 & 49 & 0.02 \\
\hline Squamous cell carcinoma & 18 & 31.6 & 39 & & 11 & 19.3 & 46 & \\
\hline Adenosquamous carcinoma & 2 & 25 & 6 & & 1 & 12.5 & 7 & \\
\hline \multicolumn{9}{|l|}{ Differentiation of tumor } \\
\hline Well & 2 & 20 & 8 & 0.94 & 0 & 0 & 10 & 0.6 \\
\hline Moderate and poor & 28 & 26.7 & 77 & & 13 & 12.4 & 92 & \\
\hline \multicolumn{9}{|l|}{ Tumor size $(\mathrm{cm})$} \\
\hline$<5$ & 18 & 23.7 & 58 & 0.5 & 8 & 10.5 & 68 & 0.76 \\
\hline$\geq 5$ & 12 & 30.8 & 27 & & 5 & 12.8 & 34 & \\
\hline \multicolumn{9}{|l|}{ Lymph node metastasis } \\
\hline Positive & 17 & 31.5 & 37 & 0.29 & 6 & 11.1 & 48 & 0.95 \\
\hline Negative & 13 & 21.3 & 48 & & 7 & 11.5 & 54 & \\
\hline \multicolumn{9}{|l|}{ Smoking status } \\
\hline No history & 12 & 25 & 36 & 0.82 & 1 & 2.1 & 47 & 0.008 \\
\hline Former or current & 18 & 26.9 & 49 & & 12 & 17.9 & 55 & \\
\hline
\end{tabular}

differentiated. Additionally, in 24 cases (42.1\%) a local lymph node invasion was detected. Of 50 cases in the ADC subgroup, $38(76 \%)$ had no history of smoking, $43(86 \%)$ had tumor dimensions of $<5 \mathrm{~cm}, 40(80 \%)$ were moderately or poorly differentiated, $28(56 \%)$ were female and $35(70 \%)$ were $<64$ years of age. The ADSCs histologically comprised ADC and SCC patterns. In this subgroup, 6 cases (75\%) were smokers, 5 cases had tumor dimensions of $<5 \mathrm{~cm}, 4$ cases were $<64$ years of age, and 4 cases exhibited lymph node metastasis.

SOX2 and MET gene amplifications in non-small-cell lung carcinoma. The amplifications of the MET and SOX2 oncogenes were detected by relative quantitative real-time PCR analysis in 115 NSCLC samples with paraffin-embedded tissues. The normalized ratios of MET and SOX2 calculated from non-cancer tissues were $0.942 \pm 0.09$ and $0.96 \pm 0.08$, respectively. Of this cohort, $11.3 \%$ (13/115) and 26.1\% (30/115) exhibited MET and SOX2 amplifications, respectively. No deletion was found in either of the genes. The patients were classified into amplified and unamplified groups and stratified by other factors (Table I, Figs. 2 and 3).

In ADCs, the 38 cases with no history of smoking were unamplified in the MET gene vs. 10 cases amplified in SOX2.
MET amplification indicated a low level in this group despite clinicopathological characteristics such as tumor dimension, lymph node invasion and age. Of the 12 smokers, 1 case was found to be amplified in MET vs. no cases in SOX2. Among 43 cases exhibiting a tumor dimension of $<5 \mathrm{~cm}, 2.3 \%$ of the cases were found to be amplified in MET and $20.9 \%$ in SOX2. Of the ADCs, 26 cases were positive for lymph node invasion and 6 cases $(23.1 \%)$ indicated SOX2 amplification vs. no cases detected in $M E T$.

In the SCC subpopulation, all 57 cases were moderately or poorly differentiated, 18 cases $(31.6 \%)$ had SOX2 amplification and 11 cases $(19.3 \%)$ had MET amplification, including 10 cases $(91 \%)$ with a history of smoking. When subdivided by the smoking status of SCCs, the amplification level of the SOX2 gene was higher than that in ADCs among smokers $\left(\chi^{2}=6.25, \mathrm{P}=0.014\right.$; below the significant level of 0.017$)$. Of the 35 cases that were younger than 64 years of age showed 1 case (2.86\%) amplified in MET and 8 (22.9\%) in SOX2 gene. From the 35 cases, MET amplification was noted in 7 cases (25\%) with a tumor dimension $<5 \mathrm{~cm}$ each, and 4 (13.8\%) with a tumor dimension $\geq 5 \mathrm{~cm}$. MET and SOX2 gene amplification were not significantly associated with tumor dimension or lymph node invasion. In this study, we demonstrated that $M E T$ 


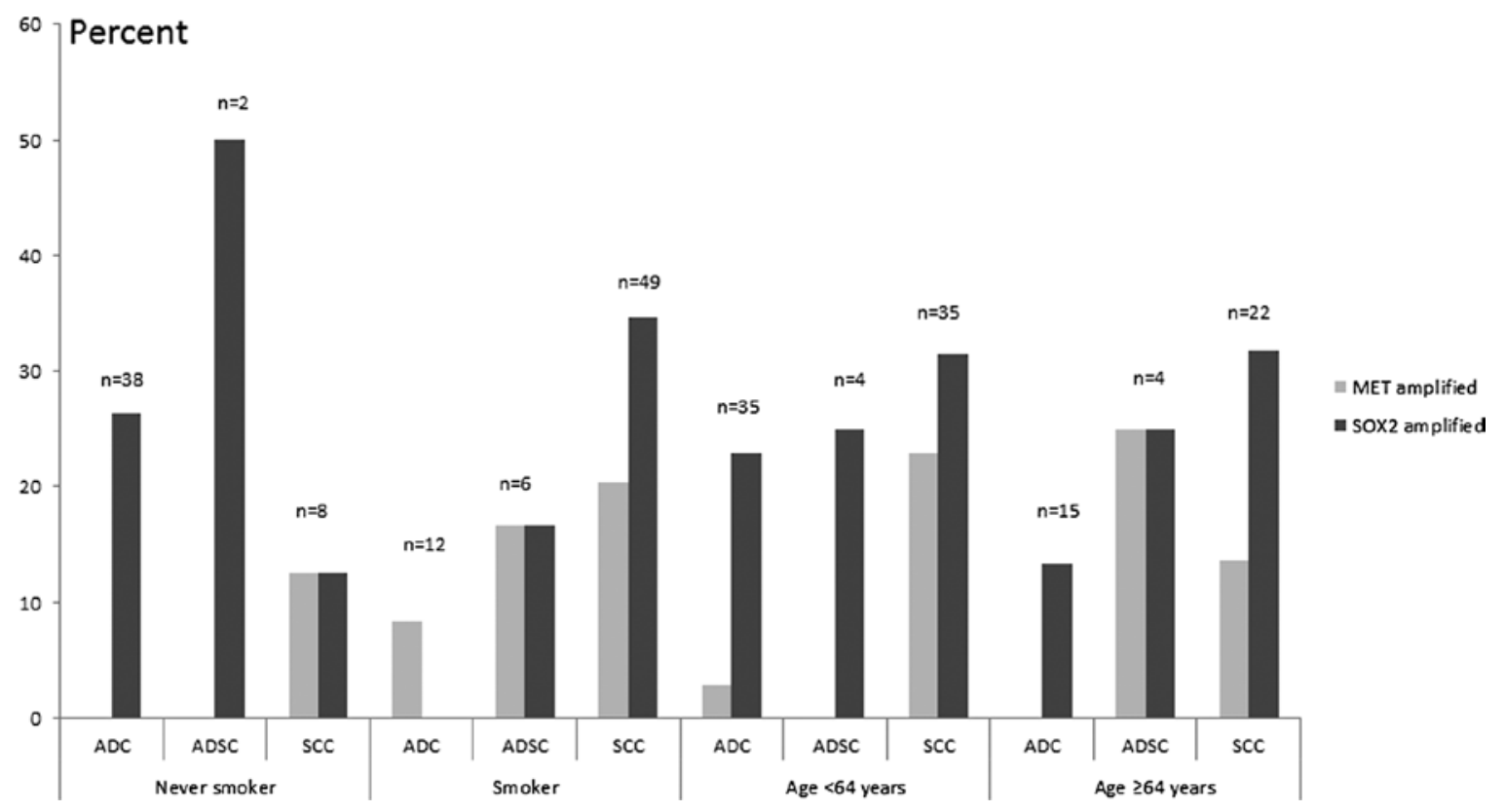

Figure 2.MET and SOX2 gene amplifications in various histological types stratified by smoking history and age. MET amplification in squamous cell carcinomas (SCCs) among patients $<64$ years of age was higher compared to adenocarcinomas (ADCs) ( $\mathrm{P}=0.03$ ). SOX2 showed preferential amplification in SCCs from smokers as compared to ADCs $(\mathrm{P}=0.014)$.

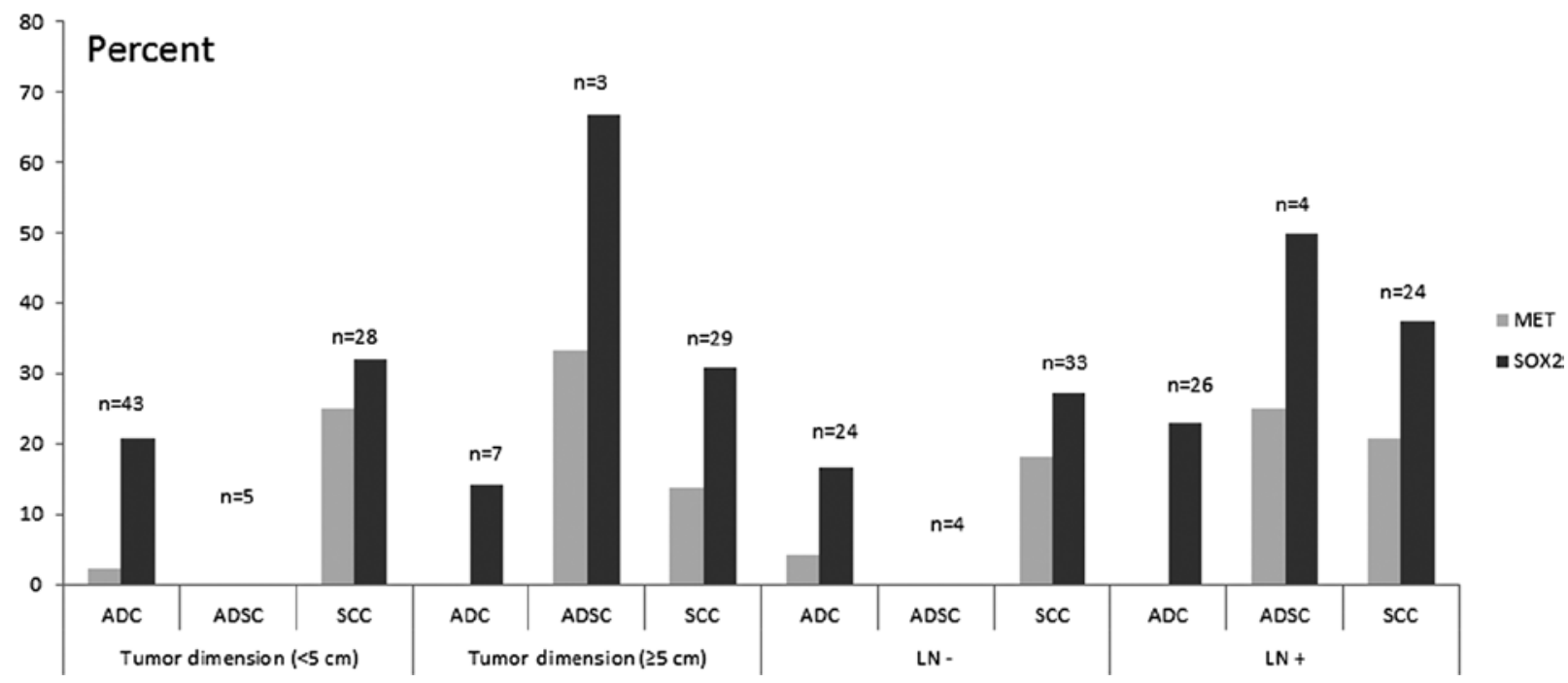

Figure 3. MET and SOX2 gene amplifications in various histological types stratified by tumor dimension and local lymph node metastasis status. Within the lymph node metastasis subgroup, $M E T$ amplification in squamous cell carcinomas (SCCs) was marginally higher than in adenocarcinomas (ADCs) ( $\mathrm{P}=0.02)$. SCCs were significantly amplified in MET compared to ADCs correlated to the tumor dimension at a smaller scale $(<5 \mathrm{~cm})(\mathrm{P}=0.01)$, whereas no significant correlation was noted when tumors were larger $(\geq 5 \mathrm{~cm}$ ). No significant difference in $S O X 2$ amplification was found related to lymph node metastasis or tumor dimension. LN: negative lymph node invasion; $\mathrm{LN}^{+}$: positive lymph node invasion.

amplification was negatively correlated with SOX2 amplification although the difference was not statistically significant $(\mathrm{r}=-0.19, \mathrm{P}>0.05)$.

In the ADSC group, the 8 cases were moderately or poorly differentiated. Of these cases, $1(12.5 \%)$ and $2(25 \%)$ exhibited MET and SOX2 amplification, respectively. Only 1 of 2 cases among non-smokers exhibited SOX2 amplification, whereas no cases exhibited MET amplification. ADSC patients younger than 64 years of age were not amplified in $M E T$ gene, and only 1 case (25\%) was amplified in SOX2 gene. Of the cases with lymph node invasion, $1(25 \%)$ and $2(50 \%)$ showed in MET and SOX2 amplification, respectively. No amplification was noted in either MET and SOX2 without lymph node metastasis (Fig. 2).

A significant difference was found in the MET amplification level among former or current smokers vs. non-smokers $\left(\chi^{2}=6.99, \mathrm{P}<0.01\right)$. SOX2 amplification occurred in $20 \%$ of ADCs, $19.3 \%$ of SCCs and $25 \%$ of ADSCs ( $\mathrm{P}=0.39)$. MET amplifications were detected in ADCs (2\%), SCCs (19.3\%) and ADSCs $(12.5 \%)\left(\chi^{2}=7.96, P=0.02\right)$. Further analysis showed that the MET level was higher in SCCs than in ADCs $\left(\chi^{2}=8.0\right.$, $\mathrm{P}=0.005$; compared under a significant level of 0.017). SOX2 
amplification was correlated with patient characteristics and tumor pathology. With the exception of a higher frequency observed in SCCs with smoking status as compared to ADCs, SOX2 amplification was not significantly associated with any other clinical or pathological variable, including gender, age, histological subtype, grade and lymph node invasion. MET amplification did not correlate with gender. Only 2 (3.51\%) SCCs with a history of smoking presented co-amplification of MET and SOX2. No co-amplification of the two genes was detected in ADCs or ADSCs. A total of 8 (22.9\%) SCCs younger than 64 years of age $(n=35)$ were correlated with amplified MET gene. However, no correlation was noted with amplified MET in ADSCs, and only 1 (2.9\%) in ADCs ( $\mathrm{P}=0.03)$ (Fig. 2). MET amplification was also detected preferentially in patients with a former or current history of smoking (17.9\%), compared with non-smokers (2.1\%). Among the patients with no history of smoking, no cases of ADCs and ADSCs had amplified $M E T$ with the exception of 1 SCC. No significant difference was noted among smokers with MET amplification in ADCs $(8.3 \%, \mathrm{n}=12)$, ADSCs $(16.7 \%, \mathrm{n}=6)$ or SCCs $(20.4 \%, \mathrm{n}=49)$ (Fig. 2). In addition, when stratified into subgroups by age and differentiation, the frequency of MET amplification in SCCs younger than 64 years of age and moderate differentiation was marginally higher than those in ADCs and ADSCs $(\mathrm{P}=0.06$, $\mathrm{n}=66$ ). MET amplification in SCCs were more frequent than in ADCs combined with clinicopathological characteristics such as younger than 64 years of age, lymph node metastasis and a tumor dimension of $<5 \mathrm{~cm}$ (Figs. 2 and 3). A total of 2 cases (20\%) of well-differentiated ADCs were detected with SOX2 amplification. In contrast, 8 cases $(20 \%)$ of ADCs, 2 cases (25\%) of ADSCs and 18 cases $(31.6 \%)$ of SCCs amplified in SOX2 with moderate and poor differentiation were detected $(\mathrm{P}=0.44)$.

MET and SOX2 amplifications were not significantly correlated whether or not they were distinguished by histological type. In ADCs discriminated by smoking status, however, it was observed that MET amplification occurred preferentially in smokers, whereas SOX2 amplification was noted in nonsmokers (Fig. 2).

\section{Discussion}

The tyrosine kinase receptor EGFR pathway has been extensively studied in NSCLC since gefitinib and erlotinib are used in NSCLC, with a clinical response more commonly observed in $\mathrm{ADC} / \mathrm{BAC}$ histology arising in non-smokers, females and patients of East Asian ethnicity (5). Recent findings have shown that MET amplification is associated with gefitinib resistance during therapy (8). On the other hand, it has been demonstrated that, using high-throughput analysis both in cell lines and in patients with lung cancer, subpopulations of cells with MET amplification existed prior to drug exposure (32). The $M E T$ gene comprises 21 exons and 20 introns $(33,34)$. The role of MET in human tumors is enhanced by mutation or amplification, leading to oncogenic changes such as cell proliferation, reduced apoptosis, angiogenesis, altered cytoskeletal function and metastasis. We found that in the subgroup with local lymph node invasion, the MET copy level in SCCs was marginally higher than in ADCs. Studies have shown that MET and its ligand HGF are mis- and over-expressed in head and neck SCC (HNSCC), resulting in the constitutive activation of the RTK system. This aberrant activity probably induces the mechanism of invasive growth by conferring an invasive potential to these tumors. $H G F$ and MET orchestrate invasive growth during the progression of HNSCC by integrating a number of independent biological responses using a specific set of signaling pathways. Specifically, $H G F / M E T$ may facilitate the detachment of neoplastic cells from primary HNSCCs through MAPK signaling, resulting in the Snail-mediated transcriptional down-regulation of E-cadherin (35). MET was also found to be deleted in ADC/BACs and SCCs; MET deletion is not a prognostic factor. However, MET appeared to be less frequently deleted compared with MET amplification (5). We identified a population with MET amplification $(11.3 \%$, 13/115) in our cohort including 11 cases of SCCs, with 10 cases being smokers. A previous study showed that the MET gene copy status was not associated with gender, smoking history, histology or stage. However, true MET amplification occurred more frequently in patients with SCC than in those with ADC. The incidence of MET amplification between SCC and ADC was significantly different (36). The clinicopathologic factors of MET amplification in NSCLC are conflicting in a number of studies. Okuda et al (37) reported that an increased MET gene copy number (GCN) was observed in $5.6 \%$ of patients with NSCLC, who were male and smokers, whereas no difference in the MET GCN status was noted with regards to histological type. In contrast, other studies observed that MET amplification (or high GCN) was not significantly associated with gender, smoking history or histology $(38,39)$. However, Go et al (36) showed that the majority of patients with true MET amplification were male smokers with SCC. Partially consistent with this study, we found that MET amplifications are not only associated with smoking status, but are more prevalent in SCCs as compared to ADCs, suggesting that MET amplification may be more involved in the oncogenesis of SCCs resulting from smoking than ADCs and may play a key role in lymph node invasion in SCC compared to ADC.

SOX2 is a key transcription factor involved in the stabilization of embryonic stem cells in a pluripotent state (40). Advances of stem cell biology have proven that both embryonic and cancer stem cells exist. Functions of such cancer stem cells include self-renewal which drives tumorigenesis, and aberrant differentiation that contributes to cellular heterogeneity. It was suggested that tumors contain a cellular population that retains key stem cell properties (41) which, in turn, have gene expression signatures closely related to embryonic stem cells (42). A high SOX2 expression was found in breast cancer (43), testicular germ cell tumors (44) and gastric adenocarcinoma (23). Expression of SOX2 protein has not been extensively studied in lung cancer. However, a recent study showed that SOX2 is strongly and diffusely expressed in approximately $90 \%$ of pulmonary SCC and $20 \%$ of ADC (45). To the best of our knowledge, this is the first report concerning high SOX2 amplification in a cohort of NSCLC. We showed that the amplification of SOX2 in SCCs and ADCs was 31.6 and $20 \%$, respectively. SOX2 is considered to be a master pluripotency controller that was recently identified as a major novel oncogene, recurrently amplified and activated in SCC $(46,47)$. These studies used a similar strategy of chromosomal aberrations screening to identify the SOX2 locus as one of the 
most frequently amplified sites over the SCC genome. They have further highlighted the recurrent $S O X 2$ activation and its indispensable role for squamous cell survival. The studies showed that SOX2 is involved in the early steps of lung SCC since it participates in transforming human bronchial epithelial cells. Furthermore, SOX2 overexpression induces the expression of the squamous markers p63 and keratin 6, indicating that SOX2 plays a role in SCC differentiation (47). However, neither study assessed the impact of the recurrent activation of SOX2 in advanced primary tumors nor how SOX2 may mechanistically be involved in tumor progression and aggressiveness. The above-mentioned studies therefore elucidate and offer novel perspectives on the multiple roles that $S O X 2$ exerts on SCC carcinogenesis.

In our study population, $89.1 \%$ (49/55) of SCC and $24 \%$ (12/50) of ADC had a history of smoking, and $34.7 \%$ (17/49) of SCCs had SOX2 amplification, whereas no amplification was found in ADCs. In contrast, 10 of 38 (26.3\%) cases involving patients with no history of smoking and with ADC presented SOX2 amplification, indicating that SOX2 amplification may be an activating pathway to ADC. Additionally, SCCs presented a higher proportion of SOX2 amplification than in ADCs and ADSCs among smokers (Fig. 2). The evidence points to the discrepancy in the oncogenesis of SCC and ADC. Comparative genomic hybridization studies have demonstrated that more than $90 \%$ of SCCs and approximately $20 \%$ of ADCs have copy number gain involving 3 q26,4, i.e., the same proportions that have been shown to have a high level of SOX2 expression at the protein level (45). Notably, these studies have found a high level of amplification only in SCC (48). These observations warrant additional studies to determine the molecular mechanisms involved in SOX2 expression in ADC. In this study, the SOX2 gene was more likely to be amplified in the subgroup of patients with no history of smoking and with ADCs. This pilot study was biased to include surgically resectable, early stage tumors. Consequently, sufficient higher-stage tumors in order to perform a well-powered statistical analysis were not available. However, we hypothesize that the SOX2 and MET genes play different roles in the carcinogenesis of SCCs and ADCs, respectively. Previous studies suggest that overexpression of SOX2 is key in the activation of the Nanog/ Oct4/SOX2 pathway, which promotes tumor cell proliferation and is associated with the short survival time of patients in stage I ADC (49). SOX2 is also activated in more advanced SCC tumors, as previously reported $(25,45)$. We conclude that SOX 2 is activated not only by overexpression but also by amplification as previously mentioned $(25,50)$. MET and SOX2 gene amplifications are more common in the SCCs of smokers. Moreover, MET amplification is intrinsic to SCCs, particularly among smokers, with regards to tumor growth, local lymph node metastasis and negative correlation with SOX2 amplification. The incidence of amplifications of MET and SOX2 is in the early stage of tumorigenesis in NSCLC. We speculate that the SOX2 gene is not only activated by amplification but is also affected by other regulators that promote its transcription, affecting its downstream genes.

ADSCs are morphologically mixed tumors comprising the two cell components ADC and SCC. To determine whether these types of tumors are a 'simple' mix of ADC and SCC or whether they present molecular specificities, as compared with the molecular characterization of the two components, Bastide et al found that genes were differentially expressed when comparing ADCs SCCs, ADSCs SCCs and ADCs ADSCs (51). Partially consistent with their findings, we observed that, when classifying the three histological subtypes, using MET and SOX2 gene amplifications that distinguished ADCs and SCCs, all ADSCs were identified as intermediate between ADCs and SCCs, with some being similar to ADCs, but others to SCCs. The results indicate that ADSCs are considered a mix of ADCs and SCCs, in various proportions. Moreover, molecular specificities were observed since we found MET and SOX2 gene amplifications among smokers in the three histological types. In conclusion, the ADSC mixed lung tumors are more complex than a 'simple' mix of ADC and SCC components. A recent study showed that neuroendocrine differentiation and ERK proliferation pathways appeared to be preferentially deregulated in ADSCs compared to ADCs and SCCs, which warrants further investigation since these pathways may partially explain the high clinical aggressiveness of ADSCs (51). Amplification of SOX2 was also found to be more preferential in ADSCs than the MET gene (Figs. 2 and 3). One limitation of our study is the insufficient number of cases. Thus, more studies should be conducted to determine the molecular specificities of ADSCs.

\section{Acknowledgements}

We thank Xue-Jing Chen, Li Zhang and Chen Zhang for their support in sample collection. Supported by the Beijing Foundation for Distinguished Scientists grant 2009D003013000001, awards from the Beijing Board of Health, China.

\section{References}

1. Travis WD, Brambilla E and Muller-Hermelink HK: World Health Organization Classification of Tumours, Pathology and Genetics: Tumours of the Lung, Pleura, Thymus and Heart. IARC Press, Lyon, 2004.

2. Laird AD and Cherrington JM: Small molecule tyrosine kinase inhibitors: clinical development of anticancer agents. Expert Opin Investig Drugs 12: 51-64, 2003.

3. Maulik G, Kijima T and Salgia R: Role of receptor tyrosine kinases in lung cancer. Methods Mol Med 74: 113-125, 2003.

4. Lamorte L and Park M: The receptor tyrosine kinases: role in cancer progression. Surg Oncol Clin N Am 10: 271-288, 2001.

5. Fukuoka M, Yano S, Giaccone G, et al: Multi-institutional randomized phase II trial of gefitinib for previously treated patients with advanced non-small-cell lung cancer (The IDEAL 1 Trial) [corrected]. J Clin Oncol 21: 2237-2246, 2003.

6. Kris MG, Natale RB, Herbst RS, et al: Efficacy of gefitinib, an inhibitor of the epidermal growth factor receptor tyrosine kinase, in symptomatic patients with non-small cell lung cancer: a randomized trial. JAMA 290: 2149-2158, 2003.

7. Shepherd FA, Rodrigues Pereira J, Ciuleanu T, et al: Erlotinib in previously treated non-small-cell lung cancer. N Engl J Med 353: 123-132, 2005.

8. Engelman JA, Zejnullahu K, Mitsudomi T, et al: MET amplification leads to gefitinib resistance in lung cancer by activating ERBB3 signaling. Science 316: 1039-1043, 2007.

9. Naldini L, Weidner KM, Vigna E, et al: Scatter factor and hepatocyte growth factor are indistinguishable ligands for the MET receptor. EMBO J 10: 2867-2878, 1991.

10. Bottaro DP, Rubin JS, Faletto DL, et al: Identification of the hepatocyte growth factor receptor as the c-met proto-oncogene product. Science 251: 802-804, 1991. 
11. Birchmeier C, Birchmeier W, Gherardi E and Vande Woude GF: Met, metastasis, motility and more. Nat Rev Mol Cell Biol 4: 915-925, 2003

12. Maulik G, Shrikhande A, Kijima T, Ma PC, Morrison PT and Salgia R: Role of the hepatocyte growth factor receptor, c-Met, in oncogenesis and potential for therapeutic inhibition. Cytokine Growth Factor Rev 13: 41-59, 2002.

13. Cooper CS, Park M, Blair DG, et al: Molecular cloning of a new transforming gene from a chemically transformed human cell line. Nature 311: 29-33, 1984

14. Chen JT, Lin TS, Chow KC, et al: Cigarette smoking induces overexpression of hepatocyte growth factor in type II pneumocytes and lung cancer cells. Am J Respir Cell Mol Biol 34 264-273, 2006.

15. Nakamura Y, Niki T, Goto A, et al: c-Met activation in lung adenocarcinoma tissues: an immunohistochemical analysis Cancer Sci 98: 1006-1013, 2007.

16. Tsao MS, Liu N, Chen JR, et al: Differential expression of Met/ hepatocyte growth factor receptor in subtypes of non-small cell lung cancers. Lung Cancer 20: 1-16, 1998.

17. Beau-Faller M, Gaub MP, Schneider A, et al: Allelic imbalance at loci containing FGFR, FGF, c-Met and HGF candidate genes in non-small cell lung cancer sub-types, implication for progression. Eur J Cancer 39: 2538-2547, 2003.

18. Smolen GA, Sordella R, Muir B, et al: Amplification of MET may identify a subset of cancers with extreme sensitivity to the selective tyrosine kinase inhibitor PHA-665752. Proc Natl Acad Sci USA 103: 2316-2321, 2006.

19. Miller CT, Lin L, Casper AM, et al: Genomic amplification of MET with boundaries within fragile site FRA7G and upregulation of MET pathways in esophageal adenocarcinoma. Oncogene 25: 409-418, 2006.

20. Que J, Luo X, Schwartz RJ and Hogan BL: Multiple roles for Sox 2 in the developing and adult mouse trachea. Development 136: 1899-1907, 2009.

21. Gontan C, de Munck A, Vermeij M, Grosveld F, Tibboel D and Rottier R: Sox 2 is important for two crucial processes in lung development: branching morphogenesis and epithelial cell differentiation. Dev Biol 317: 296-309, 2008.

22. Park KS, Wells JM, Zorn AM, et al: Transdifferentiation of ciliated cells during repair of the respiratory epithelium. Am J Respir Cell Mol Biol 34: 151-157, 2006.

23. Tsukamoto T, Mizoshita T, Mihara M, et al: Sox2 expression in human stomach adenocarcinomas with gastric and gastric-andintestinal-mixed phenotypes. Histopathology 46: 649-658, 2005

24. Li XL, Eishi Y, Bai YQ, et al: Expression of the SRY-related HMG box protein SOX2 in human gastric carcinoma. Int $\mathrm{J}$ Oncol 24: 257-263, 2004.

25. Hussenet T, Dali S, Exinger J, et al: SOX2 is an oncogene activated by recurrent 3q26.3 amplifications in human lung squamous cell carcinomas. PLoS One 5: e8960, 2010.

26. Ebright MI, Zakowski MF, Martin J, et al: Clinical pattern and pathologic stage but not histologic features predict outcome for bronchioloalveolar carcinoma. Ann Thorac Surg 74: 1640-1647, 2002.

27. Livak KJ, Flood SJ, Marmaro J, Giusti W and Deetz K Oligonucleotides with fluorescent dyes at opposite ends provide a quenched probe system useful for detecting PCR product and nucleic acid hybridization. PCR Methods Appl 4: 357-362, 1995.

28. Higuchi R, Fockler C, Dollinger G and Watson R: Kinetic PCR analysis: real-time monitoring of DNA amplification reactions. Biotechnology (NY) 11: 1026-1030, 1993.

29. Holland PM, Abramson RD, Watson R and Gelfand DH: Detection of specific polymerase chain reaction product by utilizing the 5'-3' exonuclease activity of Thermus aquaticus DNA polymerase. Proc Natl Acad Sci USA 88: 7276-7280, 1991.

30. Bieche I, Olivi M, Champeme MH, Vidaud D, Lidereau R and Vidaud M: Novel approach to quantitative polymerase chain reaction using real-time detection: application to the detection of gene amplification in breast cancer. Int J Cancer 78: 661-666, 1998.
31. Beau-Faller M, Ruppert AM, Voegeli AC, et al: MET gene copy number in non-small cell lung cancer: molecular analysis in a targeted tyrosine kinase inhibitor naive cohort. J Thorac Oncol 3: 331-339, 2008.

32. Turke AB, Zejnullahu K, Wu YL, et al: Preexistence and clonal selection of MET amplification in EGFR mutant NSCLC. Cancer Cell 17: 77-88, 2010.

33. Maestrini E, Tamagnone L, Longati $\mathrm{P}$, et al: A family of transmembrane proteins with homology to the MET-hepatocyte growth factor receptor. Proc Natl Acad Sci USA 93: 674-678, 1996.

34. Comoglio PM and Boccaccio C: The HGF receptor family: unconventional signal transducers for invasive cell growth. Genes Cells 1: 347-354, 1996.

35. De Herdt MJ and Baatenburg de Jong RJ: HGF and c-MET as potential orchestrators of invasive growth in head and neck squamous cell carcinoma. Front Biosci 13: 2516-2526, 2008.

36. Go H, Jeon YK, Park HJ, Sung SW, Seo JW and Chung DH: High MET gene copy number leads to shorter survival in patients with non-small cell lung cancer. J Thorac Oncol 5: 305-313, 2010.

37. Okuda K, Sasaki H, Yukiue H, Yano M and Fujii Y: Met gene copy number predicts the prognosis for completely resected non-small cell lung cancer. Cancer Sci 99: 2280-2285, 2008.

38. Bean J, Brennan C, Shih JY, et al: MET amplification occurs with or without T790M mutations in EGFR mutant lung tumors with acquired resistance to gefitinib or erlotinib. Proc Natl Acad Sci USA 104: 20932-20937, 2007.

39. Cappuzzo F, Marchetti A, Skokan M, et al: Increased MET gene copy number negatively affects survival of surgically resected non-small-cell lung cancer patients. J Clin Oncol 27: 1667-1674, 2009.

40. Masui S, Nakatake Y, Toyooka Y, et al: Pluripotency governed by Sox 2 via regulation of Oct $3 / 4$ expression in mouse embryonic stem cells. Nat Cell Biol 9: 625-635, 2007.

41. Wicha MS, Liu S and Dontu G: Cancer stem cells: an old idea--a paradigm shift. Cancer Res 66: 1883-1890, 2006.

42. Ben-Porath I, Thomson MW, Carey VJ, et al: An embryonic stem cell-like gene expression signature in poorly differentiated aggressive human tumors. Nat Genet 40: 499-507, 2008

43. Chen Y, Shi L, Zhang L, et al: The molecular mechanism governing the oncogenic potential of SOX2 in breast cancer. J Biol Chem 283: 17969-17978, 2008.

44. Biermann K, Heukamp LC, Steger K, et al: Genome-wide expression profiling reveals new insights into pathogenesis and progression of testicular germ cell tumors. Cancer Genomics Proteomics 4: 359-367, 2007.

45. Sholl LM, Long KB and Hornick JL: Sox 2 expression in pulmonary non-small cell and neuroendocrine carcinomas. Applied immunohistochemistry \& molecular morphology: AIMM/official publication of the Society for Applied Immunohistochemistry 18: 55-61, 2010.

46. Hussenet T and du Manoir S: SOX2 in squamous cell carcinoma: amplifying a pleiotropic oncogene along carcinogenesis. Cell Cycle 9, 1480-1486, 2010.

47. Bass AJ, Watanabe $\mathrm{H}$, Mermel $\mathrm{CH}$, et al: $\mathrm{SOX} 2$ is an amplified lineage-survival oncogene in lung and esophageal squamous cell carcinomas. Nat Genet 41: 1238-1242, 2009.

48. Bjorkqvist AM, Husgafvel-Pursiainen K, Anttila S, et al: DNA gains in $3 \mathrm{q}$ occur frequently in squamous cell carcinoma of the lung, but not in adenocarcinoma. Genes Chromosomes Cancer 22: 79-82, 1998.

49. Sholl LM, Barletta JA, Yeap BY, Chirieac LR and Hornick JL: Sox 2 protein expression is an independent poor prognostic indicator in stage I lung adenocarcinoma. Am J Surg Pathol 34: 1193-1198, 2010.

50. McCaughan F, Pole JC, Bankier AT, et al: Progressive 3q amplification consistently targets SOX2 in preinvasive squamous lung cancer. Am J Respir Crit Care Med 182: 83-91, 2010.

51. Bastide K, Ugolin N, Levalois C, Bernaudin JF and Chevillard S: Are adenosquamous lung carcinomas a simple mix of adenocarcinomas and squamous cell carcinomas, or more complex at the molecular level? Lung Cancer 68: 1-9, 2010. 